\title{
Injury-Induced Biosynthesis of Methyl-Branched Polyene Pigments in a White-Rotting Basidiomycete
}

\author{
Daniel Schwenk, ${ }^{\dagger}$ Markus Nett, ${ }^{\ddagger}$ Hans-Martin Dahse, ${ }^{\ddagger}$ Uwe Horn, ${ }^{\ddagger}$ Robert A. Blanchette, ${ }^{\S}$ \\ and Dirk Hoffmeister, ${ }^{*}$ \\ ${ }^{\dagger}$ Department of Pharmaceutical Microbiology at the Hans-Knöll-Institute, Friedrich-Schiller-Universität, Beutenbergstrasse 11a,
07745 Jena, Germany
${ }^{\ddagger}$ Leibniz Institute for Natural Product Research and Infection Biology, Hans-Knöll-Institute, Beutenbergstrasse 11a, 07745 Jena,
Germany
${ }^{\S}$ Department of Plant Pathology, University of Minnesota, 1991 Upper Buford Circle, Saint Paul, Minnesota 55108, United States
}

Supporting Information

ABSTRACT: A stereaceous basidiomycete was investigated with regard to its capacity to produce yellow pigments after physical injury of the mycelium. Two pigments were isolated from mycelial extracts, and their structures were elucidated by ESIMS and oneand two-dimensional NMR methods. The structures were identified as the previously undescribed polyenes (3Z,5E,7E,9E,11E,13Z,15E,17E)-18-methyl-19-oxoicosa$3,5,7,9,11,13,15,17$-octaenoic acid (1) and (3E,5Z,7E,9E,11E,13E,15Z,17E,19E)-20methyl-21-oxodocosa-3,5,7,9,11,13,15,17,19-nonaenoic acid (2). Stable-isotope feeding with $\left[1-{ }^{13} \mathrm{C}\right]$ acetate and $\mathrm{L}-\left[\right.$ methyl- $\left.{ }^{13} \mathrm{C}\right]$ methionine demonstrated a polyketide backbone and that the introduction of the sole methyl branch is most likely $S$-adenosyl-Lmethionine-dependent. Dose-dependent inhibition of Drosophila melanogaster larval development was observed with both polyenes in concentrations between 12.5 and 100 $\mu \mathrm{M}$. $\mathrm{GI}_{50}$ values for 1 and 2 against HUVEC (K-562 cells) were 71.6 and $17.4 \mu \mathrm{M}$ (15.4 and $1.1 \mu \mathrm{M})$, respectively, whereas $\mathrm{CC}_{50}$ values for HeLa cells were virtually identical

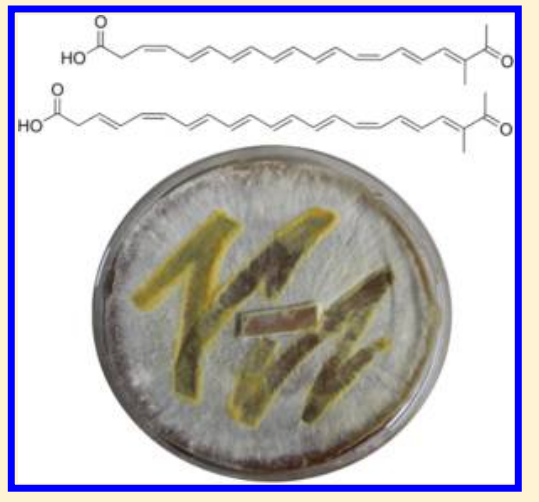
(44.1 and $45.1 \mu \mathrm{M})$.

$\mathrm{T}$ he basidiomycetes, in particular those of the subdivision agaricomycotina (the mushrooms and other "higher fungi"), are prolific producers of natural products, among them a large number of pigments whose bright colors and color changes have intrigued chemists and mycologists. ${ }^{1,2}$ Among the first accounts of natural product chemistry was the research on the color of mushroom fruiting bodies when quinones (the Ltyrosine- and L-phenylalanine-derived terphenylquinones atromentin and polyporic acid, respectively) were isolated in the late 19th century. ${ }^{3,4}$ Furthermore, since the Renaissance period, craftsmen have used wood stained by the light-insensitive fungal naphthoquinone derivative xylindein for intarsia masterpieces and parquetry. ${ }^{5}$ With regard to chemical ecology, the function of these basidiomycete pigments has largely remained elusive, ${ }^{6}$ although variegatic acid, an atromentin derivative, was suggested to play a vital role for iron redox cycling during lignocellulose disruption. ${ }^{7}$ Also, anecdotal evidence exists that basidiomycete pigments may play a role in chemical defense. Polyenes from Piptoporus australiensis are thought to deter fungivorous larvae from feeding on the fruiting bodies, ${ }^{8}$ and stephanosporin of Stephanospora caroticolor liberates 2-chloro4-nitrophenol, which exerts antimicrobial and fungicidal effects. 9

We investigated the undifferentiated mycelium of a taxonomically yet unidentified agaricomycete fungus, for which we use the preliminary code name "BY1", and its capacity to produce small-molecule natural products. Here, we describe the isolation and structural elucidation of two novel yellow methyl-branched laetiporic acid-type polyene pigments, ${ }^{10}$ whose production is inducible by physical injury and which inhibit larval development. On the basis of stable isotope-labeling we establish the polyketide origin of this polyene.

\section{RESULTS AND DISCUSSION}

Taxonomic Placement of BY1. The fungus BY1 was isolated from dead aspen wood that showed a white rot type of wood decay, and a pure axenic culture was obtained. As fruiting bodies were not available we were unable to assign a taxonomic position based on morphological characteristics. Therefore, we amplified and sequenced the internal transcribed spacer (ITS) region. A 665 bp amplicon (GenBank accession number KC514809) was used to query the NCBI database, which returned sequences with $92 \%, 91 \%$, and $90 \%$ identical nucleotides respectively from Acanthophysium lividocaeruleum, Stereum hirsutum, and Stereum rugosum (accession numbers AY618666, AF2180400, and FN539052). Sequencing of a 1750 bp portion of the large ribosomal subunit gene (accession

Received: July 8, 2014

Published: November 24, 2014 


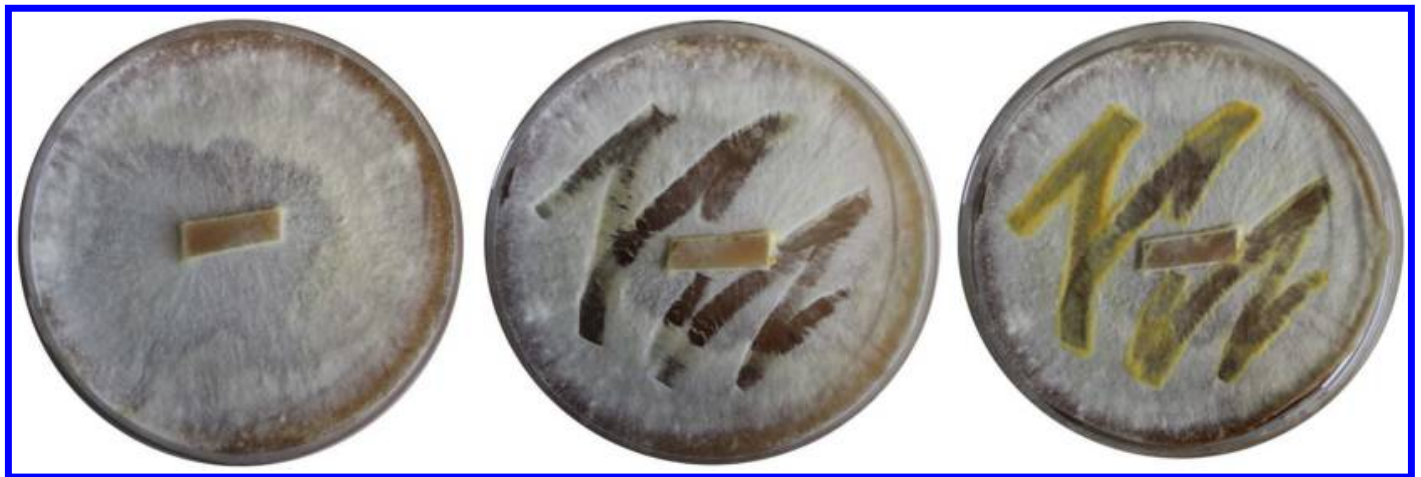

Figure 1. Cultures of BY1 grown on solid YMG medium: uninjured fungal culture (left), directly after injury (center), and mycelium 3 days past injury (right).

Table 1. NMR Spectroscopic Data for Polyenes 1 and $2^{a}$

\begin{tabular}{|c|c|c|c|c|c|c|}
\hline \multirow[b]{2}{*}{ pos. } & \multicolumn{3}{|c|}{$\begin{array}{c}(3 Z, 5 E, 7 E, 9 E, 11 E, 13 Z, 15 E, 17 E)-18 \text {-methyl-19-oxoicosa-3,5,7,9,11,13,15,17- } \\
\text { octaenoic acid (1) }\end{array}$} & \multicolumn{3}{|c|}{$\begin{array}{c}(3 E, 5 Z, 7 E, 9 E, 11 E, 13 E, 15 Z, 17 E, 19 E) \text {-20-methyl-21-oxodocosa- } \\
3,5,7,9,11,13,15,17,19 \text {-nonaenoic acid (2) }\end{array}$} \\
\hline & $\delta_{\mathrm{C}}$, type & $\delta_{\mathrm{H}},(\mathrm{J}$ in $\mathrm{Hz})$ & NOESY & $\delta_{\mathrm{C}}$, type & $\delta_{\mathrm{H}},(\mathrm{J}$ in $\mathrm{Hz})$ & NOESY \\
\hline 1 & 172.3, C & & & 172.3, C & & \\
\hline 2 & $33.5, \mathrm{CH}_{2}$ & $3.19, \mathrm{dd}(7.5,1.9)$ & $(3), 5$ & $38.2, \mathrm{CH} 2$ & 3.19 , dd $(7.4,1.2)$ & n.d. \\
\hline 3 & $125.5, \mathrm{CH}$ & $5.64, \mathrm{dt}(10.7,7.5)$ & $(2), 4$ & $128.8, \mathrm{CH}$ & $5.87, \mathrm{dt}(14.9,7.4)$ & 4 \\
\hline 4 & $131.4, \mathrm{CH}$ & 6.16 , ddd $(12.8,10.7,1.9)$ & 3,6 & 129.6, CH & 6.77 , ddd $(14.9,10.5,1.2)$ & $3,5,6$ \\
\hline 5 & $129.8, \mathrm{CH}$ & $6.58, \mathrm{t}(12.8)$ & 2,6 & 129.9, CH & $6.07, \mathrm{dd}(10.5,8.5)$ & 4,6 \\
\hline 6 & 134.8, CH & $6.38, \mathrm{~m}$ & 5 & 130.2, CH & $6.89, \mathrm{dd}(13.7,8.5)$ & $4,5,7$ \\
\hline 7 & 135.7, CH & $6.40, \mathrm{dd}(13.7,11.0)$ & 8,9 & $134.8, \mathrm{CH}$ & $6.43, \mathrm{dd}(13.7,11.3)$ & 6 \\
\hline 8 & $129.6, \mathrm{CH}$ & $6.85, \mathrm{dd}(13.7,10.6)$ & 7 & $135.8, \mathrm{CH}$ & $6.47, \mathrm{dd}(13.6,11.3)$ & 9,10 \\
\hline 9 & 132.2, $\mathrm{CH}$ & 6.13, dd $(13.7,10.6)$ & 7 & 130.0, CH & $6.93, \mathrm{dd}(13.6,10.9)$ & 8 \\
\hline 10 & 130.6, CH & 6.12 , dd $(13.7,10.0)$ & 12 & 132.3, CH & $6.19, \mathrm{~m}$ & 8 \\
\hline 11 & 131.8, CH & 6.92 , dd $(14.6,10.0)$ & 13 & $129.8, \mathrm{CH}$ & $6.94, \mathrm{dd}(14.3,10.5)$ & $-{ }^{b}$ \\
\hline 12 & $129.9, \mathrm{CH}$ & $6.88, \mathrm{dd}(14.6,10.6)$ & 10,15 & $130.6, \mathrm{CH}$ & $6.16, \mathrm{dd}(13.7,10.5)$ & 13 \\
\hline 13 & $133.8, \mathrm{CH}$ & $6.28, \mathrm{t}(10.6)$ & 11 & 131.9, CH & $6.99, \mathrm{~m}$ & 12 \\
\hline 14 & 130.4, CH & $6.21, \mathrm{~m}$ & 16 & 130.0, CH & $6.99, \mathrm{~m}$ & 17 \\
\hline 15 & 135.6, $\mathrm{CH}$ & $7.21, \mathrm{dd}(14.5,11.5)$ & 12,16 & 134.0, $\mathrm{CH}$ & 6.36 , dd $(11.1,10.6)$ & $-{ }^{b}$ \\
\hline 16 & 130.3, CH & 6.67, dd $(14.5,11.4)$ & $14,15,17,21$ & 130.6, CH & $6.28, \mathrm{dd}(11.8,11.1)$ & 17,18 \\
\hline 17 & $139.3, \mathrm{CH}$ & 7.19, dd $(11.4,1.2)$ & 16,20 & $136.0, \mathrm{CH}$ & 7.32 , dd $(14.4,11.8)$ & 14,16 \\
\hline 18 & 137.3, C & & & $130.4, \mathrm{CH}$ & 6.75 , dd $(14.4,11.3)$ & 16,23 \\
\hline 19 & 197.8, C & & & $139.8, \mathrm{CH}$ & $7.29, \mathrm{dd}(11.3,1.0)$ & 22 \\
\hline 20 & 25.7, $\mathrm{CH}_{3}$ & $2.26, \mathrm{~s}$ & 17 & 137.0, C & & \\
\hline 21 & $11.5, \mathrm{CH}_{3}$ & $1.86, \mathrm{~d}(1.2)$ & 16 & 198.8, C & & \\
\hline 22 & & & & 25.6, $\mathrm{CH}_{3}$ & $2.30, \mathrm{~s}$ & 19 \\
\hline 23 & & & & $11.5, \mathrm{CH}_{3}$ & $1.86, \mathrm{~d}(1.0)$ & 18 \\
\hline $\mathrm{OH}$ & & $10.80, \mathrm{~s}$ & n.d. & & n.d. & n.d. \\
\hline
\end{tabular}

${ }^{a}$ For spectra see Figures S5, S6, and S9-S14. ${ }^{b}$ Not assigned due to signal overlap.

number KM101461) confirmed the relationship of BY1 with the genus Stereum, as sequences with $97 \%$ identical nucleotides from Stereum spp. FCUG2671, Stereum subtomentosum, and Xylobolus frustulatus (accession numbers AF506483, AF506482, and AF506491, respectively) were returned when searching the NCBI sequence database. We therefore have assigned BY1 to the Stereaceae family within the order Russulales, a well-known group of organisms following a white-rotting lifestyle.

Isolation and Structural Elucidation of Polyene Pigments. Under standard laboratory conditions, BY1 developed a white, undifferentiated mycelium when grown on solid complex media such as yeast-malt-glucose medium. However, after being physically injured, e.g., by incision with a scalpel, the mycelium turned yellow 3 to 4 days postwounding. This pigmentation developed around at the edges of the injured site (Figure 1) and remained virtually unchanged for weeks. HPLC-DAD analysis of crude acetone extracts of BY1 mycelia, which had been injured prior to extraction, indicated the presence of two major compounds, 1 and 2. Their UV absorption maxima at $\lambda=416$ and $425 \mathrm{~nm}$, respectively (Figures S1 and S2), suggested that the metabolites might indeed contribute to the observed yellow coloration.

Compound 1 exhibited a signal at $m / z 325.1796[\mathrm{M}+\mathrm{H}]^{+}$in the high-resolution ESIMS spectrum (Figure S3), which is consistent with a molecular formula of $\mathrm{C}_{21} \mathrm{H}_{24} \mathrm{O}_{3}$ and corresponds to 10 degrees of unsaturation. The IR spectrum contained several diagnostic bands. A broad hydroxy stretching vibration at $3024 \mathrm{~cm}^{-1}$ as well as strong absorptions at 1717 and $1634 \mathrm{~cm}^{-1}$ enabled a preliminary assignment of the three oxygen atoms in $\mathbf{1}$ to a carboxylic acid function and a ketone. The latter was predicted to be conjugated with at least two double bonds due to its low vibration frequency. Additional 
Chart 1. Chemical Structures of Basidiomycete Polyenes (3Z,5E,7E,9E,11E,13Z,15E,17E)-18-Methyl-19-oxoicosa$3,5,7,9,11,13,15,17$-octaenoic acid $(1)$ and $(3 E, 5 Z, 7 E, 9 E, 11 E, 13 E, 15 Z, 17 E, 19 E)-20$-Methyl-21-oxodocosa$3,5,7,9,11,13,15,17,19-$ nonaenoic acid (2), Piptoporic Acid, and Laetiporic Acid A ${ }^{a}$

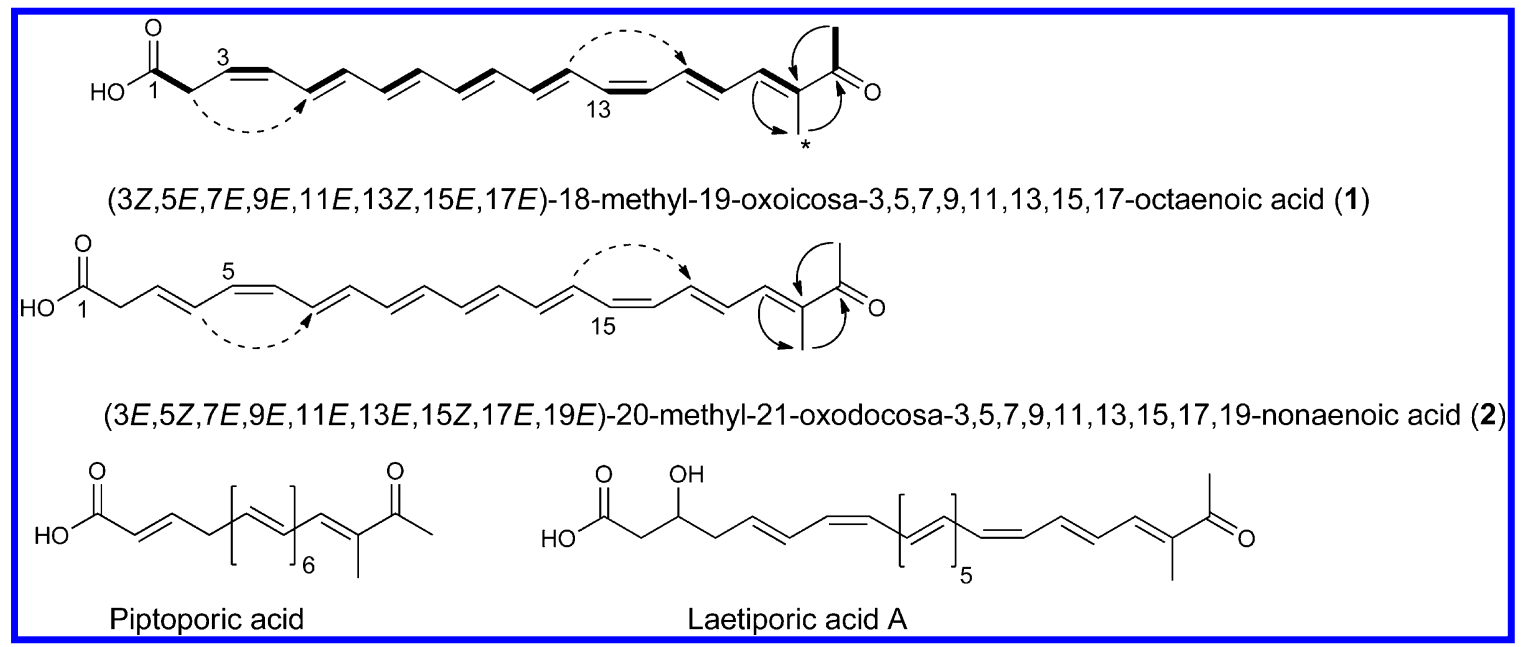

${ }^{a}$ Bold chemical bonds in 1 represent acetate units as deduced from $\left[1-{ }^{13} \mathrm{C}\right]$ acetate labeling pattern; the asterisk shows ${ }^{13} \mathrm{C}$ incorporation after feeding $1-\left[{ }^{13} \mathrm{C}\right.$-methyl $]$ methionine to cultures of BY1. Relevant HMBC (solid arrows) and NOE correlations (dashed arrows) are shown.

bands between 2000 and $1600 \mathrm{~cm}^{-1}$ supported the presence of olefinic groups, whereas alkyne or allene bands could not be detected. Furthermore, strong bending vibrations at 969 and $739 \mathrm{~cm}^{-1}$ suggested that 1 harbors both $E$ - and $Z$-configured carbon-carbon double bonds. Subsequent inspection of the ${ }^{13} \mathrm{C}$ NMR spectrum (Figure S11) confirmed the conclusions that had been drawn from the interpretation of the IR data. Two signals, which were observed at $\delta_{\mathrm{C}} 197.8$ and $172.2 \mathrm{ppm}$, were fully consistent with a ketone and a carboxylic acid. Since 16 further carbon signals could be ascribed to $\mathrm{sp}^{2}$-hybridized atoms on the basis of their chemical shifts (Table 1), the degrees of unsaturation in $\mathbf{1}$ are due to eight carbon-carbon double bonds and the two aforementioned carbonyl moieties. Only three carbon atoms of $\mathbf{1}$ are located in the aliphatic region of the ${ }^{13} \mathrm{C}$ NMR spectrum. The corresponding signals were identified as two methyl and a methylene group via a DEPT135 experiment. Afterward, all proton signals were attributed to their directly attached carbon atoms by heteronuclear singlequantum coherence (HSQC). Proton-proton correlation spectroscopy (COSY) in combination with homonuclear $J$ resolved ${ }^{1} \mathrm{H}$ NMR spectroscopy (Figures S7 and S8) and firstorder multiplet analysis revealed a continuous spin system in $\mathbf{1}$ that comprised all proton groups except for the methyl signals at $\delta_{\mathrm{H}} 2.26$ and $1.86 \mathrm{ppm}$. Heteronuclear long-range correlations from $\mathrm{H}_{2}-2$ and $\mathrm{H}-3$ to $\mathrm{C}-1$ established the position of the carboxylic acid group. Likewise, the quaternary $\mathrm{C}-18$ could be placed next to $\mathrm{CH}-17$, thereby defining the location of the last carbon-carbon double bond. On the basis of the previous assumptions, the latter had to be conjugated with the remaining ketone and, indeed, H-17 was clearly correlated with C-19 in the heteronuclear multiple-bond correlation (HMBC) spectrum. Long-range correlations from $\mathrm{H}_{3}-20$ to $\mathrm{C}-19$ and from $\mathrm{H}_{3}-21$ to $\mathrm{C}-18$ concluded the determination of the planar structure of 1 . The configuration of the double bonds was deduced from the respective ${ }^{3} J$ values and NOEs (Figure S13), respectively. On the basis of the above data, we identified compound 1 as $(3 Z, 5 E, 7 E, 9 E, 11 E, 13 Z, 15 E, 17 E)$-18-methyl-19oxoicosa-3,5,7,9,11,13,15,17-octaenoic acid (Chart 1).
Physical and spectroscopic data of $\mathbf{2}$ were similar to $\mathbf{1}$. However, a mass increase of $26 \mathrm{Da}$ was suggestive of an additional carbon-carbon double bond (Figure S4). The extension of the conjugated olefinic system was reflected by the bathochromically shifted absorbance maximum of $2(\lambda=425$ $\mathrm{nm}$ versus $416 \mathrm{~nm}$ in 1 , Figure S2). IR data confirmed the presence of a carboxylic acid and a ketone in conjugation to at least two double bonds. Bands found in the IR spectra of $\mathbf{1}$ at 969 and $739 \mathrm{~cm}^{-1}$ suggested that the structure of 2 includes both $E$ - and $Z$-configured carbon-carbon double bonds. 1D and 2D NMR data (Figures S9, S10, and S14) confirmed these findings. Assignment of proton signals and their multiplicity was accomplished in acetone- $d_{6}$. Twenty signals in the ${ }^{13} \mathrm{C}$ NMR spectrum could be attributed to $\mathrm{sp}^{2}$-hybridized atoms (Table 1; Figure S12). Interpretation of their chemical shifts indicated the presence of nine carbon-carbon double bonds, a carboxylic acid, and a ketone function. 2D NMR spectra showed the same connectivity as found for 1, except for the already anticipated extension of the olefinic chain. The two cisconfigured double bonds in 2 were deduced from ${ }^{3} J$ values and NOEs, respectively (Figure S14). Therefore, the structure of 2 was determined as $(3 E, 5 Z, 7 E, 9 E, 11 E, 13 E, 15 Z, 17 E, 19 E)-20$ methyl-21-oxodocosa-3,5,7,9,11,13,15,17,19-nonaenoic acid (Chart 1).

Among the known fungal metabolites with similar structural features are piptoporic acid (Chart 1) and related compounds ${ }^{11}$ isolated from the basidiomycete Piptoporus australiensis, and laetiporic acid A (Chart 1) and derivatives, which are the yellow pigments of the "chicken of the woods" fungus Laetiporus sulphureus. ${ }^{10,12}$ While the double bonds of piptoporic acid are all-trans configured, the major isomers of laetiporic acid $\mathrm{A}$ and 2-dehydro-3-deoxylaetiporic acid A show two and three cisconfigured double bonds, respectively. Compound $\mathbf{1}$ and piptoporic acid share the same sum formula. However, NMR data proved that these molecules are dissimilar in regard to the double-bond placement, as piptoporic acid features one double bond in conjugation with the carboxylic acid moiety that is separated from the polyene chromophore by a saturated methylene group. This results in distinct chemical shifts of the 
respective olefinic carbon atoms $\left(\delta_{\mathrm{C}}=121.8\right.$ and $147.8 \mathrm{ppm}$ for piptoporic acid). ${ }^{11}$ However, these signals were not detected in the spectra of 1 and 2.

Stable-Isotope Labeling and Biosynthetic Considerations. To determine the biosynthetic origin of BY1 polyenes, 1 was purified from cultures containing stable-isotope-labeled substrates. Feeding of both $\left[1-{ }^{13} \mathrm{C}\right]$ acetate and $\mathrm{L}-\left[\right.$ methyl- $\left.{ }^{13} \mathrm{C}\right]$ methionine resulted in incorporation and increased ${ }^{13} \mathrm{C}$ NMR signal intensity of individual carbon atoms (Figures S15 and S16). When 1 was isolated from cultures fed with L[methyl- $\left.{ }^{13} \mathrm{C}\right]$ methionine, only the signal corresponding to the methyl group residing at C-21 was enhanced in the ${ }^{13} \mathrm{C}$ NMR spectrum (Figure S16). In contrast, the signal pattern resulting from $\left[1-{ }^{13} \mathrm{C}\right]$ acetate feeding showed an increase for every other carbon atom of 1 (Figure S15), beginning with the carboxylic acid functionality (C-1, Chart 1 ) and extending throughout the entire linear main chain. These results prove a polyketidic biosynthesis, in which the identified formal acetate units correspond to the canonical building blocks acetyl-CoA (starter unit) and malonyl-CoA (extender unit). Moreover, the increased signal intensity of C-21 shows the S-adenosyl-Lmethionine (SAM)-dependent introduction of this methyl group, most likely by $C$-methyltransferase activity, which is frequently integral to fungal reducing polyketide synthases. ${ }^{13,14}$ Intriguingly, both 1 and 2 exclusively feature $\beta, \gamma$-positioned double bonds within the formal acetate units.

Following the biosynthetic logic of polyketide synthesis, shifted double bonds represent a rare exception to the standard pattern of polyketide natural products where double bonds are found between the formal acetate units (i.e., $\alpha, \beta$-positioned to the acetate carbonyl function). Beyond the laetiporic acid/ piptoporic acid family of basidiomycete compounds, precedence for shifted polyketide double bonds also stems from research on the antitumor compound ansamitocin, which is produced by Actinosynnema pretiosum. Taft et al. (2009) proposed a mechanism in which a vinylogous syn-elimination by a dehydratase domain in the third module of the asm polyketide synthase mediates the double-bond migration. ${ }^{15}$ Specifically, a tetraketide intermediate oriented in a specific, required conformation within the catalytic center prevents the canonical $\alpha, \beta$-syn-elimination. A second well-documented example of shifted polyketide double bonds pertains to the formation of the shifted diene system in rhizoxin D1, a biosynthetic intermediate en route to rhizoxin, which is made by the endosymbiotic bacterium Burkholderia rhizoxinica. ${ }^{16}$ The double bonds introduced at positions 9 and 11 are shifted sequentially by two different dehydratase domains. Mechanistically, the first double-bond migration takes place analogously to the ansamitocin mode, whereas the subsequent second shift is most likely carried out by a dedicated module intrinsic to the rhizoxin polyketide synthase.

Anti-insect Activity of Polyenes 1 and 2. As polyene production was induced by physically injured mycelium (Figure 1), we hypothesized that $\mathbf{1}$ and $\mathbf{2}$ might be bioactive molecules and play a role in fungal defense, e.g., against fungivores. A report on a possible role of piptoporic acid to protect Piptoporus australiensis carpophores from flies feeding on fruiting bodies ${ }^{8}$ prompted us to perform bioactivity tests on the insect model Drosophila melanogaster. Further support for our hypothesis comes from earlier studies that showed that certain fungal pigments reduce the pupation rate of $D$. melanogaster. ${ }^{17}$ However, this study did not include polyene structures. To expose all stages of the $D$. melanogaster life cycle to polyenes $\mathbf{1}$ and $\mathbf{2}$, adult female flies were kept on either untreated or polyene-amended medium. After 7 days, the number of pupating larvae was determined and the pupation number was determined relative to controls on polyene-free medium (Figure 2). Both $\mathbf{1}$ and $\mathbf{2}$ impacted the development of

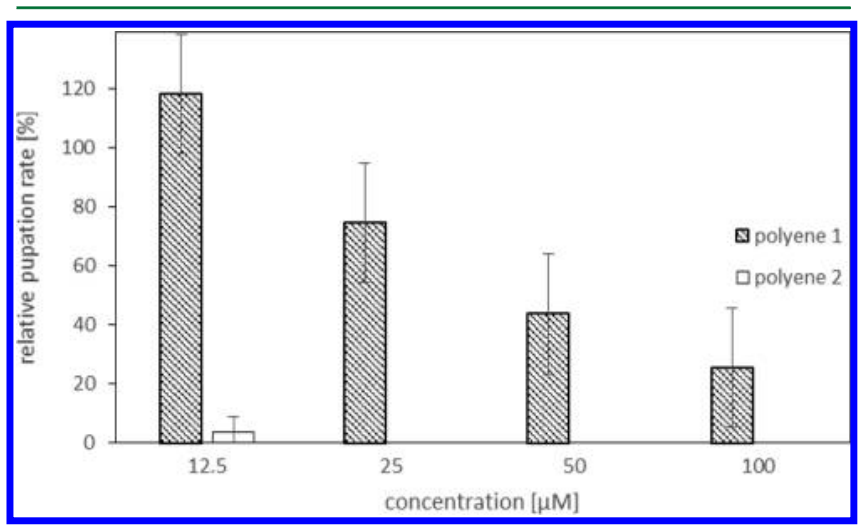

Figure 2. Relative pupation rate of Drosophila melanogaster progeny with respect to concentrations of polyenes $\mathbf{1}$ and $\mathbf{2}$ in the medium. Control pupation levels were determined on unmodified medium and normalized to $100 \%$.

D. melanogaster, although they differed in their respective efficacy. While 1 did not reduce the number of pupating larvae at $12.5 \mu \mathrm{M}$, a clear dose-dependent inhibition at concentrations up to $100 \mu \mathrm{M}$ was observed ( 5 versus 18 in controls). When exposed to $12.5 \mu \mathrm{M} \mathrm{2}$, only a minimum number of pupating larvae were observed (two animals in one of the vessels). At higher doses, pupation was not observed at all.

Injury-elicited chemical conversion of inactive precursor molecules into bioactive compounds has been reported for various basidiomycetes. In fruiting bodies of Mycena galopus, saponification of benzoxepine esters to the respective bioactive alcohols in response to wounding of mycelium was found. ${ }^{18}$ Stearoylvelutinal represents the inactive ester found in numerous species within the Russulales, from which the unstable sesquiterpene velutinal is liberated after injury and subsequently transformed to aldehyde and alcohol derivatives that confer a pungent taste to the fruiting body. ${ }^{19}$ The corticioid basidiomycete Aleurodiscus amorphus, which also belongs to the Russulales, uses the cyanohydrine ether aleurodisconitrile, from which hydrocyanic acid is oxidatively released immediately following injury. ${ }^{20}$ The above defense strategies were shown for fruiting bodies and rely on rapid activation of the toxic compound in order to respond promptly to fungivory. In contrast, our results on the stereaceous fungus BY1 pertain to undifferentiated mycelium and suggest a slow response taking days. Although we cannot exclude a release from a colorless precursor, the delayed onset of polyene appearance in the mycelium suggests that $\mathbf{1}$ and $\mathbf{2}$ are synthesized de novo following injury. Production of these polyenes seems to aim at a sustained and long-term protective effect. Our results support a role in protecting the mycelium from feeding larvae. However, the underlying mechanism remains elusive. A direct toxic effect of the polyene pigments 1 and $\mathbf{2}$ on D. melanogaster larvae appears possible, thus preventing further development. Alternatively, a repellent effect of polyenes may prevent larvae from feeding, thus leading to starvation and, ultimately, death. For two reasons, a deterring effect of $\mathbf{1}$ and $\mathbf{2}$ appears less likely: first, when larvae were exposed to $12.5 \mu \mathrm{M}$ of pigment 1 , orange-colored larvae were 
observed (Figure S17), which is highly suggestive of a pigment uptake. Second, insects, and specifically D. melanogaster, evaluate food sources primarily based on intricate olfactory cues, $^{21}$ which would favor volatiles serving as repelling compounds. This was shown for naphthalenes produced by the endophytic fungus Muscodor vitigenus, which repel the wheat stem sawfly, Cephus cinctus. ${ }^{22}$ Neither $\mathbf{1}$ nor $\mathbf{2}$ is volatile. However, they may represent components of a multicompound repellent/protection system.

Bioactivity against Human Cell Lines. We investigated the effect of $\mathbf{1}$ and $\mathbf{2}$ on HUVEC (human umbilical vein epithelial cells) and K-562 leukemia cells in antiproliferative assays. $\mathrm{GI}_{50}$ values for $\mathbf{1}$ and 2 were 71.6 and $17.4 \mu \mathrm{M}$ against HUVEC and 15.4 and $1.1 \mu \mathrm{M}$ against K-562 cells (Figures S18 and S19). The pronounced difference in $\mathrm{GI}_{50}$ values between HUVEC and K-562 cell lines suggests a stronger effect on faster dividing cells, and the latter value is comparable with those of clinically used drugs that were tested in parallel in the same assay. For irinotecan, tamoxifen, and idarubicin, $\mathrm{GI}_{50}$ values of $1.9,5.2$, and $3.3 \mu \mathrm{M}$ were found for $\mathrm{K}-562$ cells, respectively. Both polyene pigments exerted equal cytotoxicity on HeLa cells $\left(\mathrm{CC}_{50}=44.1\right.$ and $45.1 \mu \mathrm{M}$ for 1 and 2, respectively, Figure S20).

Research on the structurally related falconensones A and B regarding their inhibitory effect on the HL60 leukemia cell line and other cancer cell lines in vitro and in vivo suggests that the 1-methyl-2-oxo-1-propylidene terminus, in conjunction with a conjugated olefinic system, favors cytotoxic effects on mammalian cell lines. ${ }^{23,24}$ This structural feature is shared by all members of the laetiporic/piptoporic acid family of natural products. However, other structural elements are required for activity, and our results point to chain length being relevant for cytotoxic properties, as the chain-extended polyene $\mathbf{2}$ is more active than $\mathbf{1}$.

Basidiomycete polyenes have been credited with protecting mycelium from fungivory; however they have not been described in the context of an inducible defense. The polyenes $\mathbf{1}$ and $\mathbf{2}$ are novel members of the laetiporic acid/piptoporic acid family of basidiomycete pigments, which is diversified by the new compounds in that all double bonds are shifted. Our work shows inducible pigment production by injury but only very slow depigmentation, as well as inhibition of development of the model organism D. melanogaster. Taken together, these results suggest a role of the identified polyenes in protection against insect fungivores over extended periods of time.

\section{EXPERIMENTAL SECTION}

General Experimental Procedures. To record UV-vis spectra, compounds were dissolved in methanol. Spectra were acquired on a ScanDrop instrument (Analytik Jena) using Winaspect software (version 2.4). IR spectra were measured on a Jasco FT/IR 4100 instrument. 1D and 2D NMR spectra of polyenes 1 and 2 were recorded in THF- $d_{8}$ and acetone- $d_{6}$, respectively, at $300 \mathrm{~K}$, and chemical shifts were referenced relative to internal residual nondeuterated solvent traces (THF: $\delta_{\mathrm{H}} 1.72 \mathrm{ppm}, \delta_{\mathrm{C}} 25.3 \mathrm{ppm}$; acetone: $\delta_{\mathrm{H}} 2.04 \mathrm{ppm}, \delta_{\mathrm{C}} 29.8 \mathrm{ppm}$ ). NMR spectra were recorded on Bruker Avance III spectrometers. HRESIMS data were generated on an Exactive Orbitrap instrument (Thermo Scientific) using the direct injection port. For preparative HPLC an Agilent 1260 series instrument equipped with a Phenomenex Luna $\mathrm{C}_{18}$ column $(250 \times$ $21.2 \mathrm{~mm}, 10 \mu \mathrm{m}$ particle size) was used. Analytical HPLC was performed on an Agilent 1200 instrument fitted with a Zorbax Eclipse XDB $C_{18}$ column $(150 \times 4.6 \mathrm{~mm}, 3.5 \mu \mathrm{m}$ particle size $)$. All chromatograms were recorded at $\lambda=420 \mathrm{~nm}$; the respective diode array detectors covered the wavelength range $\lambda=190-600 \mathrm{~nm}$. Chemicals, solvents, stable-isotope-labeled compounds, and media components were from Cortecnet, Deutero, Eurisotop, Roth, SigmaAldrich, and VWR. Oligonucleotide primers were synthesized by Eurofins MWG Operon; DNA sequencing (Sanger) was performed by GATC.

Fungal Strain and Fermentation Conditions. The BY1 isolate was cultured from aspen wood located at the University of Minnesota Cloquet Forestry Station located in northern Minnesota, and isolation procedures were previously reported. ${ }^{25}$ The culture has been deposited at the Jena Microbial Resource Collection (JMRC) under the registration number SF:011241. The fungus was maintained and cultivated on YMG agar (4 g/L D-glucose, $4 \mathrm{~g} / \mathrm{L}$ yeast extract, $10 \mathrm{~g} / \mathrm{L}$ malt extract, $18 \mathrm{~g} / \mathrm{L}$ agar). Growth was at room temperature in the dark, for approximately 2 weeks. To induce pigment formation, the mycelium was injured using a scalpel and incubated for another 3-4 d, followed by harvest of the mycelium for compound extraction. Stable isotope feeding experiments were carried out essentially as described above, but with a total culture volume of $2 \mathrm{~L}$ (100 Petri dishes). The ${ }^{13} \mathrm{C}$-label was supplied by adding either filter-sterilized sodium $\left[1-{ }^{13} \mathrm{C}\right]$ acetate $\left(12.2 \mathrm{mM}\right.$ final) or $\mathrm{L}$-[methyl $\left.{ }^{13} \mathrm{C}\right]$ methionine $(3.35$ $\mathrm{mM}$ final) to YMG medium prior to pouring the Petri dishes. To extract genomic DNA from mycelium, BY1 was grown in liquid YMG medium as a stationary culture for 2 weeks at room temperature in the dark.

Polyene Isolation and Analysis. Polyene isolation from mycelium was carried out on ice and, whenever possible, protected from light. Pigment-producing mycelium was frozen at $-20{ }^{\circ} \mathrm{C}$, crushed, and immediately exhaustively extracted by vigorous shaking with acetone. The resulting organic phase was passed through a cellulose filter and subsequently transferred into brown glass flasks. The acetone was evaporated in a rotary evaporator at room temperature. The impure and water-insoluble compounds precipitated in residual water and were collected by centrifugation for $5 \mathrm{~min}$ at $3220 \mathrm{~g}$ at $4{ }^{\circ} \mathrm{C}$. After decanting the water, the samples were stored at $-80{ }^{\circ} \mathrm{C}$ and dissolved in methanol for chromatographic purification. Pure compounds were obtained by preparative HPLC running a water/acetonitrile $(\mathrm{ACN})$ solvent system. The gradient began at $60 \%$ $\mathrm{ACN}$ and was linearly increased to $100 \% \mathrm{ACN}$ over $20 \mathrm{~min}$ at a flow rate of $21 \mathrm{~mL} / \mathrm{min}$. Fractions containing single compounds were pooled. The acetonitrile was evaporated, and the polyenes were precipitated in the aqueous phase and collected as described above.

(3Z,5E,7E, 9E, 11E, 13Z,15E,17E)-18-Methyl-19-oxoicosa$3,5,7,9,11,13,15,17$-octaenoic acid (1): amorphous, orange solid; UV $(\mathrm{MeOH}) \lambda_{\max } 259(\log \varepsilon=4.08)$ and $416(\log \varepsilon=4.37) \mathrm{nm} ; \mathrm{IR}_{\text {(neat) }}$ 3024 (b), 1717 (s), 1634 (s), 1388 (s), 1236 (s), 996 (s), 969 (s), 796 (s), and $739(\mathrm{~s}) \mathrm{cm}^{-1} ;{ }^{1} \mathrm{H}$ and ${ }^{13} \mathrm{C}$ NMR data (THF-d, $d_{8}, 500$ and 125 $\mathrm{MHz}$, respectively), see Table 1; HRESIMS $m / z 325.1796[\mathrm{M}+\mathrm{H}]^{+}$, calcd for $\mathrm{C}_{21} \mathrm{H}_{25} \mathrm{O}_{3} 325.1798$.

(3E, 5Z,7E, 9E, $11 E, 13 E, 15 Z, 17 E, 19 E)$-20-Methyl-21-oxodocosa$3,5,7,9,11,13,15,17,19-$ nonaenoic acid (2): amorphous, orange solid; $\mathrm{UV}(\mathrm{MeOH}) \lambda_{\max } 276(\log \varepsilon=4.10)$ and $425(\log \varepsilon=4.53) \mathrm{nm}$; $\mathrm{IR}_{\text {(neat) }} 3024(\mathrm{~b}), 1721(\mathrm{~s}), 1641$ (s), $1236(\mathrm{~s}), 996(\mathrm{~s}), 968(\mathrm{~s}), 796$ $(\mathrm{s})$, and $739(\mathrm{~s}) \mathrm{cm}^{-1} ;{ }^{1} \mathrm{H}$ and ${ }^{13} \mathrm{C}$ NMR data (acetone- $d_{6}, 600$ and $150 \mathrm{MHz}$, respectively), see Table 1; HRESIMS $m / z 351.1953[\mathrm{M}+$ $\mathrm{H}]^{+}$, calcd for $\mathrm{C}_{23} \mathrm{H}_{27} \mathrm{O}_{3} 351.1955$.

Nucleic Acid Isolation and Analysis of the Internal Transcribed Spacer Region. A $100 \mu \mathrm{g}$ amount of BY1 mycelium, grown for 2 weeks in liquid medium (see above), was snap-frozen in liquid nitrogen and lyophilized overnight. Isolation of genomic DNA was done essentially following a described procedure, ${ }^{26}$ but modified in that the phenol-chloroform wash step was repeated twice. The internal transcribed spacer regions 1 and 2 were amplified using ITS1 and ITS4 primers and large ribosomal subunit (LSU) gene by primers $5.8 \mathrm{~S}-\mathrm{R}$ and LR7, following published protocols. ${ }^{27,28}$ The nucleotide sequences of the BY1 ITS region and the partial LSU gene have been deposited at GenBank under accession numbers KC514809 and KM101461, respectively.

Bioactivity Assays. HUVEC and K-562 cell lines were used in antiproliferation assays, and HeLa cells were used to determine 
cytotoxic activity, according to described protocols. ${ }^{29}$ Substances tested were dissolved in dimethyl sulfoxide, which was also used as control. Inhibitory activity against D. melanogaster was essentially determined as described. ${ }^{17}$ Three female $D$. melanogaster WT specimens (Canton-S strain, adult stage, 6 days of age) were transferred into plastic vessels, each containing $20 \mathrm{~mL}$ of nutrition medium (118 $\mathrm{g} / \mathrm{L}$ treacle, $11 \mathrm{~g} / \mathrm{L}$ brewer's yeast, $4.1 \mathrm{~g} / \mathrm{L}$ agar, $95 \mathrm{~g} / \mathrm{L}$ maize meal, 2.4 $\mathrm{g} / \mathrm{L}$ propionic acid, $3.3 \mathrm{~g} / \mathrm{L}$ nipagin $30 \%)$ either with or without compounds 1 and $2(12.5,25,50$, and $100 \mu \mathrm{M}$; added prior to solidification of the medium; all experimental setups as biological triplicate). All vessels were kept at $25^{\circ} \mathrm{C}$ in a moist atmosphere. Fly development was examined under a dissecting microscope to assess development and to quantitate Drosophila progeny. After $7 \mathrm{~d}$, the number of larvae in the puparium stage was determined and pupation rate was calculated relative to untreated controls.

Table 2. Biological Activity of Polyenes 1 and 2 against Human Cell Lines

\begin{tabular}{|c|c|c|c|}
\hline & \multicolumn{2}{|c|}{ antiproliferative effect } & \multirow{2}{*}{$\frac{\text { cytotoxicity }}{\text { HeLa }}$} \\
\hline & HUVEC & K-562 & \\
\hline & $\mathrm{GI}_{50}[\mu \mathrm{M}]^{a}$ & $\mathrm{GI}_{50}[\mu \mathrm{M}]$ & $\mathrm{CC}_{50}[\mu \mathrm{M}]^{b}$ \\
\hline 1 & 71.6 & 15.4 & 44.1 \\
\hline 2 & 17.4 & 1.1 & 45.1 \\
\hline
\end{tabular}

${ }^{a} \mathrm{GI}_{50}$ (growth inhibition): concentration that causes $50 \%$ of maximum inhibition of cell proliferation. ${ }^{b} \mathrm{CC}_{50}$ (cytotoxicity concentration): concentration that causes cell death of $50 \%$ of host cells.

\section{ASSOCIATED CONTENT}

\section{S Supporting Information}

UV-vis, 1D and 2D NMR spectra, a photograph of larvae coloration, and bioactivity data of $\mathbf{1}$ and 2 . This material is available free of charge via the Internet at http://pubs.acs.org.

\section{AUTHOR INFORMATION}

\section{Corresponding Author}

*Phone: +49 3641 949850. Fax: +49 3641 949852. E-mail: dirk. hoffmeister@hki-jena.de.

\section{Notes}

The authors declare no competing financial interest.

\section{ACKNOWLEDGMENTS}

D.S. gratefully acknowledges a doctoral fellowship by the International Leibniz Research School for Microbial and Biomolecular Interactions (ILRS Mibintact). We thank A. Perner and $\mathrm{H}$. Heinecke (Hans-Knöll-Institute Jena) for recording HRMS and NMR spectra, respectively. We also thank Dr. M. Knaden (Max Planck Institute for Chemical Ecology, Jena) for supporting Drosophila melanogaster bioassays.

\section{REFERENCES}

(1) Zhou, Z.-Y.; Liu, J.-K. Nat. Prod. Rep. 2010, 27, 1531-1570.

(2) Jiang, M.-Y.; Feng, T.; Liu, J.-K. Nat. Prod. Rep. 2011, 28, 783808.

(3) Stahlschmidt, C. Liebigs Ann. Chem. 1877, 187, 177.

(4) Thörner, W. Ber. Dtsch. Chem. Ges. 1878, 11, 533-535.

(5) Blanchette, R.; Wilmering, A. M.; Baumeister, M. Holzforschung 1992, 46, 225-232.

(6) Spiteller, P. Chem.-Eur. J. 2008, 14, 9100-9110.

(7) Eastwood, D.; Floudas, D.; Binder, M.; Majcherczyk, A.; Schneider, P.; Aerts, A.; Asiegbu, F. O.; Baker, S. E.; Barry, K.; Bendiksby, M.; Blumentritt, M.; Coutinho, P. M.; Cullen, D.; De Vries,
R. P.; Gathman, A.; Goodell, B.; Henrissat, B.; Ihrmark, K.; Kauserud, H.; Kohler, A.; LaButti, K.; Lapidus, A.; Lavin, J. L.; Lee, J. H.; Lindquist, E.; Lilly, W.; Lucas, S.; Morin, E.; Murat, C.; Oguiza, J. A.; Park, J.; Pisabarro, A. G.; Riley, R.; Rosling, A.; Salamov, A.; Schmidt, O.; Schmutz, J.; Skrede, I.; Stenlid, J.; Wiebenga, A.; Xie, X.; Kües, U.; Hibbett, D. S.; Hoffmeister, D.; Högberg, N.; Martin, F.; Grigoriev, I. V.; Watkinson, S. C. Science 2011, 333, 762-765.

(8) Gill, M.; Steglich, W. Prog. Chem. Org. Nat. Prod. 1987, 51, 1317.

(9) Lang, M.; Spiteller, P.; Hellwig, V.; Steglich, W. Angew. Chem., Int. Ed. 2001, 40, 1704-1705.

(10) Weber, R. W. S.; Mucci, A.; Davoli, P. Tetrahedron Lett. 2004, 45, 1075-1078.

(11) Gill, M. J. Chem. Soc., Perkin Trans. 1 1982, 1449-1453.

(12) Davoli, P.; Mucci, A.; Schenetti, L.; Weber, R. W. Phytochemistry 2005, 66, 817-823.

(13) Song, Z.; Cox, R. J.; Lazarus, C. M.; Simpson, T. J. ChemBioChem 2004, 5, 1196-1203.

(14) Elliott, C. E.; Callahan, D. L.; Schwenk, D.; Nett, M.; Hoffmeister, D.; Howlett, B. J. Fungal Genet. Biol. 2013, 53, 50-58.

(15) Taft, F.; Brunjes, M.; Knobloch, T.; Floss, H. G.; Kirschning, A. J. Am. Chem. Soc. 2009, 131, 3812-3813.

(16) Kusebauch, B.; Busch, B.; Scherlach, K.; Roth, M.; Hertweck, C. Angew. Chem., Int. Ed. 2010, 49, 1460-1464.

(17) Besl, H.; Blumreisinger, M. Z. Mykol. 1983, 49, 165-170.

(18) Peters, S.; Jaeger, R. J.; Spiteller, P. Eur. J. Org. Chem. 2008, $1187-1194$

(19) Sterner, O.; Bergman, R.; Kihlberg, J.; Wickberg, B. J. Nat. Prod. 1985, 48, 279-288.

(20) Kindler, B. L.; Spiteller, P. Angew. Chem., Int. Ed. 2007, 46, 8076-8078.

(21) Knaden, M.; Strutz, A.; Ahsan, J.; Sachse, S.; Hansson, B. S. Cell Rep. 2012, 1, 392-399.

(22) Daisy, B. H.; Strobel, G. A.; Castillo, U.; Ezra, D.; Sears, J.; Weaver, D. K.; Runyon, J. B. Microbiology 2002, 148, 3737-3741.

(23) Takahashi, N.; Iwahori, A.; Kawai, K.-I.; Fukui, T. Arch. Biochem. Biophys. 1998, 360, 113-120.

(24) Tamagawa, K.; Shimizu, K.; Ebine, T.; Maitani, Y.; Fukui, T.; Kawai, K.-I.; Takahashi, N. Clin. Cancer Res. 2001, 7, 3551-3558.

(25) White, W. J. Biology and control of blue stain fungi in Populus tremuloides. Master Thesis, University of Minnesota, Saint Paul, MN, 1996; p 73.

(26) Lee, B. S.; Taylor, J. W. In PCR Protocols: A Guide to Methods and Applications; Innis, M. A.; Gelfand, D. H.; Sninsky, J. J.; White, T. J., Eds.; Academic Press, Inc.: New York, 1990; pp 282-287.

(27) White, T. J.; Bruns, T.; Lee, S.; Taylor, J. W. In PCR Protocols: A Guide to Methods and Applications; Innis, M. A.; Gelfand, D. H.; Sninsky, J. J.; White, T. J., Eds.; Academic Press, Inc.: New York, 1990; pp 315-322.

(28) Vilgalys, R.; Hester, M. J. Bacteriol. 1990, 172, 4238-4246.

(29) Krauth, F.; Dahse, H.-M.; Rüttinger, H.-H.; Frohberg, P. Bioorg. Med. Chem. 2010, 18, 1816-1821. 